\title{
Revealing the Weak Interaction Mechanism of Crystalline Cellulose I $\alpha$ by Molecular Dynamics Simulations *
}

\author{
Hong-Hui Zhang ${ }^{\mathrm{a}, \mathrm{b}}$, Xue-Wei Jiang a,b,* \\ ${ }^{a}$ Wuhan Textile and Apparel Digital Engineering Technology Research Center, School of Fashion, \\ Wuhan Textile University, No. 1 Fangzhi Road, Wuhan, Hubei, China \\ ${ }^{\mathrm{b}}$ Hubei Key Laboratory of Biomass Fibers and Eco-dyeing \& Finishing, Wuhan Textile University, No. \\ 1 Sunshine Avenue, Jiangxia District, Wuhan, Hubei, China
}

\begin{abstract}
According to our previous works on cellulose $\mathrm{I} \beta$ and $\mathrm{I} \alpha$, the weak interactions, though easily ignored, play certain role in the stability mechanism of nature cellulose. These weak interactions should never be ignored or underestimated. In this work, a molecular dynamics study of cellulose I $\alpha$ was reported to evaluate the weak interactions under various temperatures. The polar and non-polar solvation interactions and hydrogen bonding were taken into account. The Van der Waals, electrostatic, polar solvation and non-polar solvation energy per chain were estimated up to $-131.68,-56.38,29.16$ and $-41.76 \mathrm{Kcal} / \mathrm{mol}$ at room temperature. The weak interactions behaviors of cellulose I $\alpha$, including that of the cellulose $\mathrm{I} \beta$ and $\mathrm{I} \alpha$ reported previously, were compared. The results indicate that hydrogen bonding contribute obviously for the intrachain stability. The interchain electrostatic interaction maintain a reasonable level under $300 \mathrm{~K}$ but decreases rapidly with the ascending of temperature. The polar and non-polar solvation interaction plays an important role not only to interchain under high temperature but also to the intersheet stability. In addition, the hydrogen bonding in intersheet is weaker than that of intrachain and interchain. The result is same as cellulose $\mathrm{I} \beta$ that relatively weak hydrogen bonding and strong nonbonded interactions keep the intersheet stability collaboratively.
\end{abstract}

Keywords: Weak Interaction; Cellulose I $\alpha$; Molecular Dynamics; Hydrogen Bonding

\section{Introduction}

Serving as reinforcement of wood, glass, cotton, and ramie and so on, cellulose is widely recognized as a sustainable source for every trade $[1,2]$. Cellulose is a linear polysaccharide, and always forms with high crystallinity ranging from 1000 to 10000 [3]. Because of its abundance and

\footnotetext{
*Project supported by the Scientific Research Project of Hubei Provincial Department of Education (Q20141604) and Laboratory of Biomolecular Dynamics, Wuhan Institute of Physics and Mathematics, Chinese Academy of Sciences for computing supporting.

${ }^{*}$ Corresponding author.

Email address: xwjiang@wtu.edu.cn (Xue-Wei Jiang).
} 
sustainability, cellulose has been under investigation since early years of polymer science [49]. Natural cellulose occurs as two different crystal phases, $\mathrm{I} \alpha$ and $\mathrm{I} \beta[4,10]$. Cellulose $\mathrm{I} \alpha$ is predominant in bacterial, algae, and fungi, whereas higher plants have a bigger percentage of cellulose $\mathrm{I} \beta$ [11]. According to the $\mathrm{CP} / \mathrm{MAS}$ NMR spectra recording, the proportion of cellulose I $\alpha$ was estimated to be 60-70 percent for Acetobater cellulose, 65 for valonia cellulose, and 60-70 percent I $\beta$ within cotton fibres $[4,12]$. Since then, a considerable part of cellulose research was devoted to decipher the atomic structure of the dimers. Roman and infrared spectroscopy was employed to uncover the meticulous details of a cellulose structure and it was discovered that cellulose I $\alpha$ was less thermally stable than $\mathrm{I} \beta[3,13,14]$. Nishiyama et laid down the basement of more accurate assessment of potential structure, and accurate positions of all atoms and hydrogenbonding network were revealed with Synchrotron X-ray and electron diffraction methodology [15, 16]. The fine structure of native crystalline has important implications for understanding of physical properties within many other cellulose-based materials.

For the existence of the dimorphism, the thorough understanding of native cellulose requires the determination of both two, not only one. Therefore, the study of cellulose I $\alpha$ is a favorable supplement of native cellulose. Various improved models were proposed to elucidate the hydrogen binding networks and molecular arrangement [3,17], and Nishiyama et proposed the re-orientation including the cell parameters and dihedrals of cellulose I $\alpha$ [16]. According to the electron microscopy research, Cellulose $\mathrm{I} \alpha$ has a triclinic structure with one cellobiose in each unit cell of $\mathrm{P} 1$ symmetry, where $\mathrm{a}=6.717 \AA, \mathrm{b}=5.962 \AA, \mathrm{c}=10.4 \AA$ (chain direction), $\alpha=118.08^{\circ}$, $\beta=114.8^{\circ}, \gamma=80.37$, while cellulose $\mathrm{I} \beta$ crystallizes with a two-chains monoclinic unit of $\mathrm{P} 2{ }_{1}$ symmetry $[15,16]$. Constituted by stacking cellulose sheets, cellulose I $\alpha$ has linear chains along the $\mathrm{c}$ direction and the repeat unit has different glucose monomers. Hydrogen bonding bindings are widely perceived as the major reason for the stable structure of native cellulose. The most frequently occupied hydrogen bonds are intrachain $\mathrm{O}_{2} \mathrm{HO} 2 \cdots \mathrm{O} 6$ ', O3HO $3 \cdots \mathrm{O} 5$ ' and interchain O6HO6... O3'. All of those are identified as the primary effect factors to keep chains rigid by Heiner et [18]. The hydrogen bonding interaction has been estimated that between 4.06 and $7.17 \mathrm{Kcal} / \mathrm{mol}$ [16]. Hydrogen bonding is experimentally most demonstrative, but insufficient to elucidate solvent resistance [19]. It is essential to reveal the intricate interplay of the relevant molecular interactions.

There is not much work focused on the weak interactions in cellulose before. French evaluated the energies of cellulose I with the MM3 force field, and found that the lattice energies is the major difference between celluloce I, while the lattice energies are mostly consituted with Van der Waals interaction [20]. The models they used were not based on high resolution experimental results. Heiner attributes the stability of cellulose I to intermolecular Coulomb interactions [18]. Our previous work shows that electrostatic interaction also play a certain role in cellulose I $\alpha$ and $\mathrm{I} \beta[21,22]$. The nonbonded interactions polar solvation and non-polar solvation should also be taken into account to acquire more comprehensive knowledge of the interaction network in native cellulose. The GLAYCAM 06 force field has been shown to be reasonably reliable in the prediction of structures and energies of saccharide [21,23]. GLYCAM 06 force field is a universal force field for biological macromolecules designed and developed by KIRSCHNER et al [24]. GLYCAM06 force field uses hydroxyl van der Waals parameter compatible with Amber, and fits the electrostatic potential energy calculated by quantum mechanics, so the GLYCAM 06 force field can express the interaction better between molecules. Considering the relationship between partial charge and molecular conformation, GLYCAM06 can reduce the influence of intersheet hydrogen bonding on cell parameter, then more reasonable cell parameters can be accuqed with 
this force field [24].

\section{Molecular Dynamic Simulation}

The crystallographic model, presented in Fig. 1 was used in all-atom molecular dynamic simulations. Cellulose chains stack together to form elementary cellulose fibrils, then consistitute the crystal structure. The cell parameters are as follows: $a=6.717 \AA, b=5.962 \AA, c=10.400 \AA$, $\alpha=118.08^{\circ}, \beta=114.80^{\circ}, \gamma=80.37^{\circ}[16]$. In higher plants such as cotton fibers, elementary cellulose fibers are aggregated into rosette-shaped bundles. Generally, the structure of cellulose bundles is composed of 5-48 glucose chains. Referring to the previous work of molecular modeling, the model was constructed with 36 chains. The degree of polymerization of each chain was 20 [25].

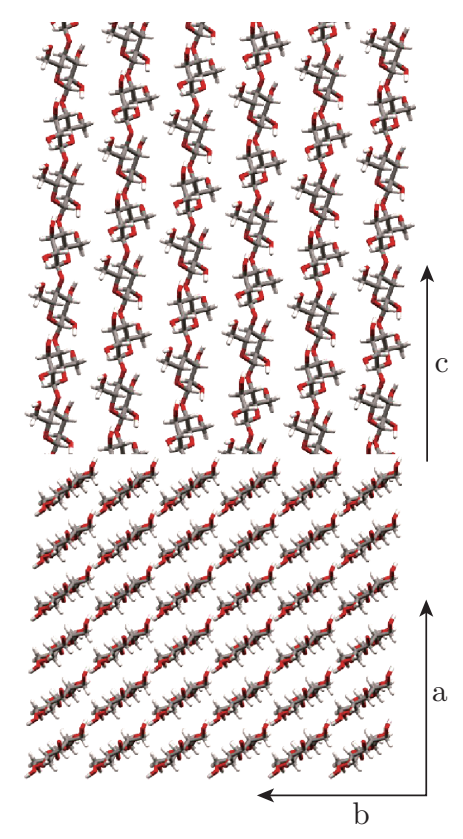

Fig. 1: Structure of cellulose I $\alpha$ and the definition of direction a, b, c

The molecular dynamics simulations are adopted to investigate the crystallization in water solution under the conditions of various temperatures $(300 \mathrm{~K}, 350 \mathrm{~K}, 400 \mathrm{~K}, 450 \mathrm{~K}, 500 \mathrm{~K}$ and $550 \mathrm{~K}$ ). All molecular simulations were performed with the package Amber. The crystalline fibril was parametrized by GLYCAM-06 force field. The microfibril was immersed in TIP3P water, with at least $12 \AA$ thickness in all directions. The solution system was optimized and then equilibrated by NVT MD (constant numbers of particles, volume and temperature). Then NPT MD was carried out to implement the equilibration and achieve the atmospheric pressure of 1 bar. With total pressure fixed, density of cellulose changed and remained almost constant after equilibration. The time step was 2 fs. The long-range electrostatic interactions were treated by the particle mesh Ewald method. All bond distance involving hydrogen atoms were constrained by SHAKE. The Langevin thermostat controlled the temperature. Non-bonded interactions were truncated at $12 \AA$. The production runs were carried out in the NPT ensemble. Berendsen barostat was used to control system pressure. 10 ps was selected as pressure relaxation time and the compressibility was $1 \times 10^{-6} \mathrm{bar}^{-1}$. The simulations lasted for $100 \mathrm{~ns}$, with the atomic 
coordinates collected per 10 ps. The change of temperature and total energy in every system over simulation progressing is presented in Fig. 2. No sudden downs or ups appear, suggesting that the dynamic simulations are relatively equilibrium. The interactions were calculated by the post-processing program MMPBSA.py. Pairwise interactions decomposed with 1-4 terms were added to internal potential terms. The average values of each chain are taken from every part of 36 chains:

$$
E=\frac{\sum_{r} \sum_{k} E_{j k}}{n}
$$

where the $r$ represents the chain number, and $k, j$ are the residue numbers. There $\mathrm{n}$ is the total chain number, 36. $E$ is the total energy (Van der Waals, electrostatic, polar solution, non-polar solution interactions).
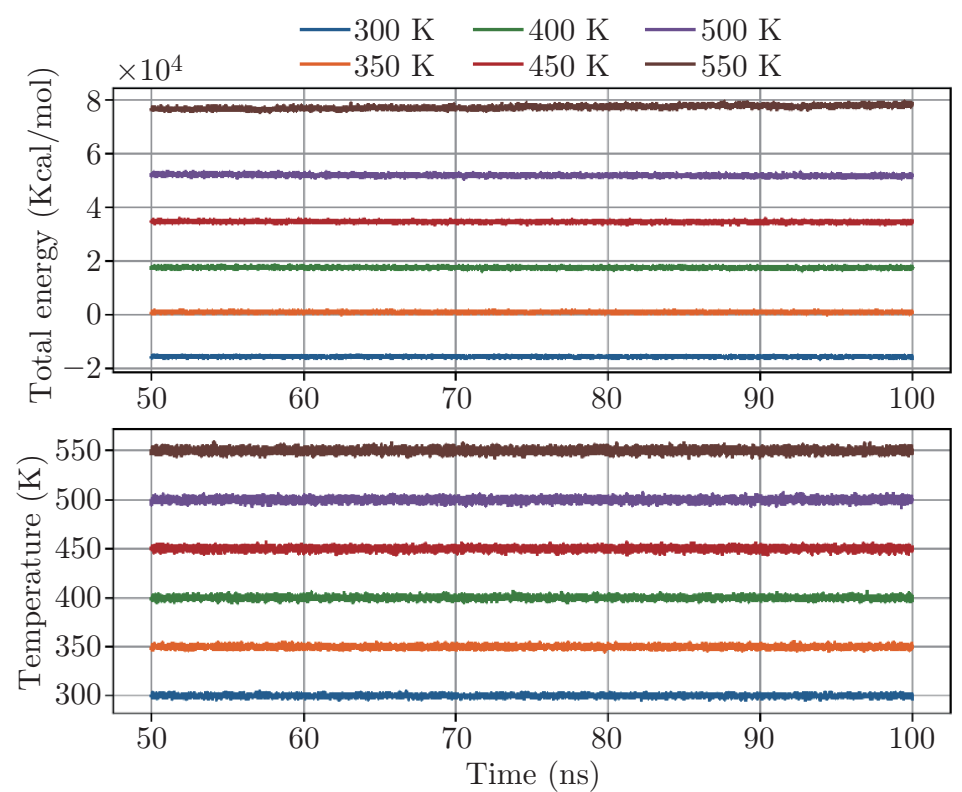

Fig. 2: The change of temperature and total energy under different systems

\section{$3 \quad$ Results and Discussions}

Different from previous works made on cellulose $\mathrm{I} \beta$ and $\mathrm{I} \alpha$, the polar solvation and nonpolar solvation energy were taken as the research topic $[21,22]$. The interaction are constituted by four parts: internal energy, Van der Waals, electrostatic and solvation energy. The results show that internal energy keeps the value at zero, so the other three parts are considered to influence the stability of cellulose I $\alpha$. It can be concluded that Van der Waals force is much more important in balancing the structure at high temperature than electrostatic interaction and Van der Waals also makes more devotion in interchain and intersheet when temperature ascends in the paper presented in TBIS [22]. In this work, solavtion energy is taken into consideration to probe for more through understanding of weak interaction mechanism in cellulose I $\alpha$. Solvation energy is the interaction between a solvent and molecules or ions of a solute [26]. The solute is the compound that is going to be dissolved in the solvent. When immersed in polar solvent, polar solvation energy is presented, otherwise the nonpolar solvation appearing. Solvation energy is responsible for many 
properties of a solute. Such as stability, solubility or reactivity. The following is organized in four parts: firstly, the whole impression is given about the whole interaction per chain. And then, the whole is splitted into three sections-intrachain, interchain, and intersheet. The definition of intrachain, interchain and intersheet is shown in Fig. 3. Besides, the hydrogen bonding network was also analysed to elucidate the mechanism quantitatively. The weak interaction mechanisms of intrachain, interchain, intersheet are described respectively.
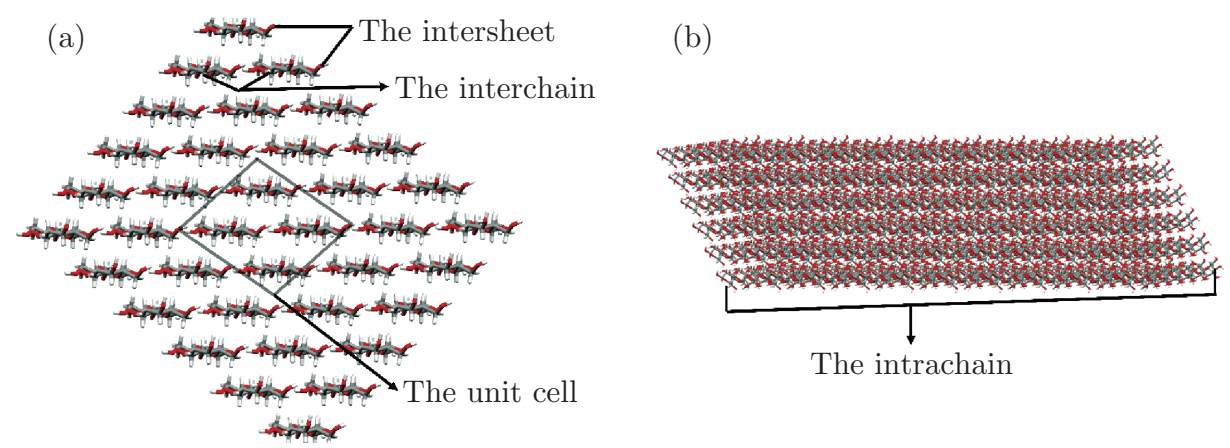

Fig. 3: The schematic diagram of cellulose I $\alpha$. a: The ab plane of cellulose $\mathrm{I} \alpha$ and the definition of the unit cell, the intersheet, the interchain. b: The 3D model of cellulose along c direction and the definition of the intrachain

\subsection{The Weak Interaction Per Chain}

Fig. 4 shows the variation trend of nonbonded interactions per chain in cellulose I $\alpha$ under different tempratures.The Van der Waals, electrostatic, polar solvation, and non-polar solvation interactions per chain are $-131.68,-56.38,29.16,-41.76 \mathrm{Kcal} / \mathrm{mol}$ in $300 \mathrm{~K}$. The results means that Van der Waals, electrostatic and non-polar solvation interactions appear to be attraction forces, while polar solvation energy is the repulsion force. The results showed that the average Van der Waals and electrostatic interactions per glucose $(-6.58,-2.82 \mathrm{Kcal} / \mathrm{mol})$ were in good accordance with those of Heiner $(-6.48,-2.75 \mathrm{Kcal} / \mathrm{mol})[18]$. Compared with previous work made in cellulose $\mathrm{I} \alpha$, Van der Waals interaction is beyond other nonbonded interactions, and solvation energy keep the same order of magnitude with electrostatic interaction [22]. The situation is same as that of cellulose $\mathrm{I} \beta$, in which the Van der Waals interaction is higher than electrostatic and solvation energy. Besides, according to the research on cellulose $\mathrm{I} \beta$, the solvation and electrostatic energy play a major role in intersheet [21]. Therefore, polar and non-polar solvation energy need to be analysed to see what roles they play in the stability of cellulose I $\alpha$. In order to present the changes of energy more intuitively, the absolute values were taken to draw graphics below. According to Fig. 4, the polar and non-polar solvation interactions keep the same trend with that of Van der Waals and electrostatic energy, for they all decrease with the increase of temperature [22]. Compared with the situation in room temperature, the rate of decrease of each interaction is $5.42 \%, 31.11 \%, 13.65 \%, 4.57 \%$ respectively at $550 \mathrm{~K}$, which means under high temperature, the electrostatic interaction decreases most obviously, followed by polar solvation energy. In other words, overall, Van der Waals and non-polar solvation interactions contribute significantly to the thermal stability of cellulose $\mathrm{I} \alpha$. So the study of solvation energy is important not only to the structural stability of cellulose but also the thermal stability.

In order to further verify the relationship between the interactions and temperature, one-way ANOVA test was conducted. One-way ANOVA method is used to test whether the same factors 


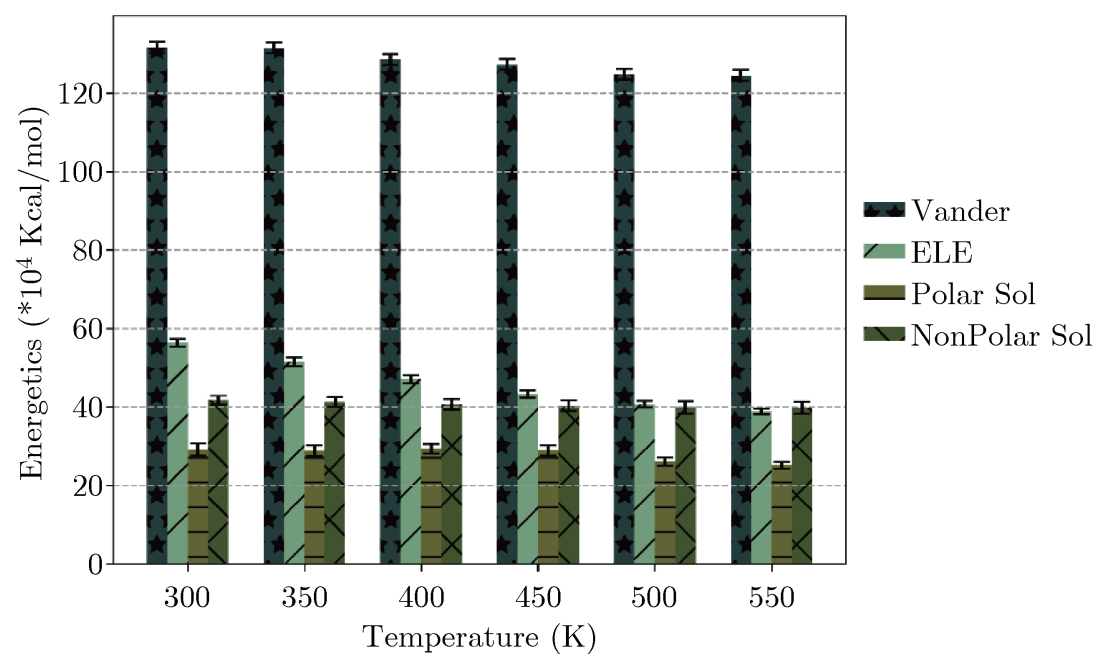

Fig. 4: The average non-bonded interactions per chain

at different levels affect the outcome variables, also known as F-test. The influencing factors there are temperatures. The magnitude of temperature represents different levels. The result variables are Van der Waals, electrostatic, polar and non-polar solvation interactions. According to the oneway ANOVA test result in Table 1, the F values of one-way ANOVA test are 3.869, 73.69, 48.32 and 5.11, respectively. The significant coefficients are 0.002, 0.000, 0.000 and 0.000 , which are less than 0.05. Therefore, it can be deduced that there may be different weak interactions mechanism under different temperatures. In addition, many scientists believe that hydrogen bonding is the main stabilizing factor. Our previous work in cellulose $\mathrm{I} \beta$ have concluded that hydrogen bonding dominates in intrasheet, while Van der Waals, electrostatic and solvation interactions are of significance in intersheet [21]. But whether the same situation is happenning in cellulose I $\alpha$ or not deserves our attention. The quantitave analysis of hydrogen bonding is also needed to understand the weak interaction mechanism. Based on the experimental data, it is assumed that there may be a synergistic effect between weak hydrogen bonding and nonbonded interactions in intersheet, which jointly maintains the intersheet stability of cellulose I $\alpha$ and makes it difficult to hydrolyze [16, 27-29]. Therefore, hydrogen bonding was also taken into considerition in this work.

Table 1: The ANOVA test of nonbonded interactions under different temperatures

\begin{tabular}{lcc}
\hline & $\mathrm{F}$ & $\mathrm{P}$ \\
\hline Van der Waals & 3.869 & 0.002 \\
electrostatic & 73.69 & 0.000 \\
Polar solvation & 48.32 & 0.000 \\
non-polar solvation & 5.11 & 0.000 \\
\hline
\end{tabular}

The calculation of hydrogen bonding is restricted by several conditions. The restriction conditions are that the distance between two oxygen atoms is less than $3.5 \AA$, and the angle between donor-hydrogen acceptor atom is greater than $150^{\circ}$. The trend of change in average occupancy and numbers of hydrogen bonding is shown in Table 2. Taking the occupancy at $300 \mathrm{~K}$ as the reference, the hydrogen bonding occupancy at $350-550 \mathrm{~K}$ is lower than that at room temperature. 
That is to say, the occurrence frequency of hydrogen bonding decreases with the increase of temperature. The reduction rates of hydrogen bond occupancy were $6.1 \%, 20.97 \%, 15.52 \%, 28.65 \%$, $26.25 \%$ respectively. This indicates that the hydrogen bonding is not sensitive to temperature change in the range of 300-350 K, but the occupancy decreases significantly at $400 \mathrm{~K}$, which is obviously different from the reduction ratio of hydrogen bond less than $15 \%$ at $400 \mathrm{~K}$ in cellulose $\mathrm{I} \beta$ [30]. That is to say, the hydrogen bond network of cellulose I $\alpha$ changes dramatically around $400 \mathrm{~K}$, and the transition temperature is lower than that of cellulose $\mathrm{I} \beta$ [30]. And the number of hydrogen bonds in cellulose $\mathrm{I} \alpha$ does not decrease with the increase of temperature. The numbers of hydrogen bonds under 300-550 K are 1 784, 1 793,1 784, 1825, 1903 and 1788 respectively. The effect of temperature on the number of hydrogen bonds are not obvious, but for cellulose $\mathrm{I} \beta$, the opposite law appears $[31,32]$. With the increase of temperature, the hydrogen bonds in cellulose I $\alpha$ may be broken, and a considerable number of hydrogen bonds were formed but relatively weaker. Therefore, although the hydrogen bond occupancy was inversely proportional to temperature, there was no significant difference in the number of hydrogen bonds.

Table 2: The average occupancy and numbers of hydrogen bonding under different temperature

\begin{tabular}{ccccccc}
\hline & $300 \mathrm{~K}$ & $350 \mathrm{~K}$ & $400 \mathrm{~K}$ & $450 \mathrm{~K}$ & $500 \mathrm{~K}$ & $550 \mathrm{~K}$ \\
\hline average occupancy & 0.7839 & 0.7298 & 0.6195 & 0.6622 & 0.5593 & 0.5781 \\
The number of hydrogen bonding & 1784 & 1793 & 1784 & 1825 & 1903 & 1788 \\
\hline
\end{tabular}

\subsection{The Weak Interactions in Intrachain}

Distribution of intrachain nonbonded interactions and change of intrachain hydrogen bonding of cellulose I $\alpha$ are presented in Fig. 5(a) and Table 3. Seen from the Fig. 5(a), at room temperature, the Van der Waals, electrostatic and solvation interactions are obviously minimal. The mean value of polar solvation energy is highest of those four weak interactions with the mean value

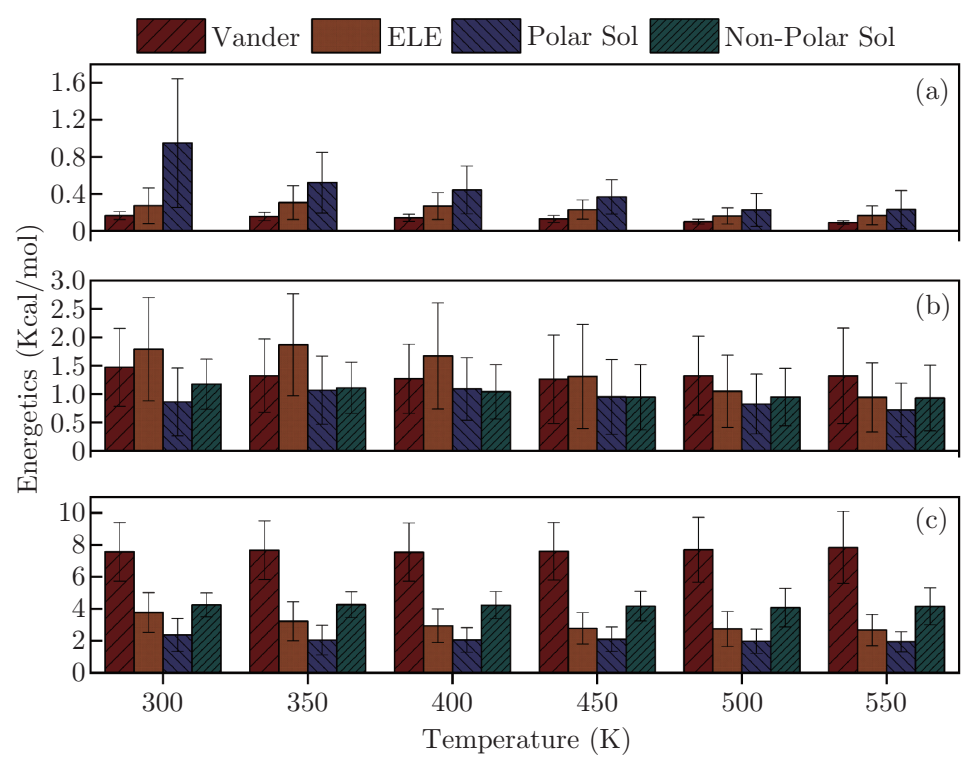

Fig. 5: The distribution of nonbonded interactions in (a) intrachain, (b) interchain, (c) intersheet 
Table 3: The average occupancy and numbers of intrachain hydrogen bonding under different temperature

\begin{tabular}{ccccccc}
\hline & $300 \mathrm{~K}$ & $350 \mathrm{~K}$ & $400 \mathrm{~K}$ & $450 \mathrm{~K}$ & $500 \mathrm{~K}$ & $550 \mathrm{~K}$ \\
\hline average occupancy & 0.8463 & 0.8018 & 0.7149 & 0.7273 & 0.653 & 0.6703 \\
The number of hydrogen bonding & 1108 & 1019 & 811 & 712 & 730 & 691 \\
\hline
\end{tabular}

being $0.9491 \mathrm{Kcal} / \mathrm{mol}$. The non-polar solvent energy is close to 0 . From our previous findings, the intrachain Van der Waals and electrostatic interactions are minimal and weaker than the intrachain polar solvation, and those interactions are obviously tiny compared with cellulose I $\beta$ $[21,22]$. For cellulose $\mathrm{I} \beta$, when the temperature is below $400 \mathrm{k}$, the electrostatic interaction of intrachain has some contributes to the stability of cellulose. Under high temperature, the electrostatic has teeny contribution to the thermal stability just as Van der Waals, and solvation energy has little contribution to the stability of cellulose [21]. So, the intrachain interactions are weak in cellulose $\mathrm{I} \alpha$ and $\mathrm{I} \beta$. The intrachain energetics of cellulose $\mathrm{I} \alpha$ decreases with increasing temperature. At $550 \mathrm{~K}$, the polar solvation, electrostatic and Van der Waals interactions are $0.2313,0.1674$ and $0.0912 \mathrm{Kcal} / \mathrm{mol}$, respectively [22]. Under the same force field, the interaction of cellulose $\mathrm{I} \beta$ shows different changing rules. Van der Waals interaction in cellulose $\mathrm{I} \beta$ increases firstly, then decreases under 500-550 K, and electrostatic energy decreases significantly. Solvent energy decreases as a whole [21]. From those comparison with the work made on cellulose I $\alpha$ and $\mathrm{I} \beta$, it is found that intrachain interactions are all small and there must be some other factor to keep the balance of intrachain structure [21, 22].

According to Table 2, although the intrachain non-bond interactions of cellulose I $\alpha$ is relatively weak, its hydrogen bond occupancy is obviously high. The average hydrogen bond occupancy at various temperatures is $0.8463,0.8018,0.7149,0.7273,0.653$ and 0.6703 , which are higher than the overall hydrogen bond occupancy, showing a downward trend with the increase of temperature. The number of hydrogen bonds decreases gradually. At $300 \mathrm{~K}$, the number of hydrogen bonds is 1 108. At $550 \mathrm{~K}$, the number of hydrogen bonds is 691. It is inferred that new hydrogen bonding network has been formed and there must be some fracture between intrachain hydrogen bonding.

\subsection{The Weak Interactions in Interchain}

Distribution of interchain nonbonded interactions and change of interchain hydrogen bonding of cellulose I $\alpha$ are presented in Fig. 5(b) and Table 4. At room temperature, the electrostatic interaction has more contribution than Van der Waals and solvation energy. The average value of electrostatic interaction is $1.7933 \mathrm{Kcal} / \mathrm{mol}$, followed by Van der waals, non-polar and polar solvation interactions with the average values being $1.4712,1.1779$ and $0.8628 \mathrm{Kcal} / \mathrm{mol}$, respectively [22]. The interchain electrostatic interaction in cellulose $\mathrm{I} \beta$ is also stronger than Van der Waals force and solvent energy [21]. With the increase of temperature, the interchain Van der Waals and polar solvation erergy of cellulose I $\alpha$ increase firstly and then decrease [22]. The change is relatively stable. The electrostatic interaction decreases as a whole. At $550 \mathrm{~K}$, the electrostatic interaction decreases to $52.55 \%$ compared to room temperature [22]. The nonpolar solvation energy is inversely proportional to the temperature change. The mean values of Van der Waals, electrostatic, polar solvation, nonpolar solvation interactions at $550 \mathrm{~K}$ are 1.3237 , $0.9423,0.9308,0.7216 \mathrm{Kcal} / \mathrm{mol}$ [22]. So under high temperature Van der Waals interaction 
is more important than electrostatic, polar solvation, nonpolar solvation interactions, while the structural stability contribution of electrostatic interaction in cellulose $\mathrm{I} \beta$ is always the highest than Van der Waals and solvation interactions [21].

The interchain nonbonded interactions of cellulose I $\alpha$ is stronger than that of intrachain, however, from Table 4, the occupancy of interchain hydrogen bond is weaker than that intrachain. The average interchain hydrogen bond occupancy at different temperatures is $0.7413,0.6898$, 0.596, $0.6687,0.558,0.5507$, respectively. At $400 \mathrm{~K}$, the interchain hydrogen bond occupancy decreases sharply. The number of hydrogen bonds increased gradually. At $300 \mathrm{~K}$, there are 577 hydrgen bondings. At $550 \mathrm{~K}$, the number of hydrogen bondsis 738 . So there must be some fraction in strong hydrogen bonding and formation of relatively weak ones. Meanwhile, the synergistic effect can be concluded because the hydrogen bond complements with Van der Waals, electrostatics, polar solvation and non-polar solvation interactions.

Table 4: The average occupancy and numbers of interchain hydrogen bonding under different temperature

\begin{tabular}{ccccccc}
\hline & $300 \mathrm{~K}$ & $350 \mathrm{~K}$ & $400 \mathrm{~K}$ & $450 \mathrm{~K}$ & $500 \mathrm{~K}$ & $550 \mathrm{~K}$ \\
\hline average occupancy & 0.7413 & 0.6898 & 0.5960 & 0.6687 & 0.5580 & 0.5507 \\
The number of hydrogen bonding & 577 & 645 & 671 & 729 & 745 & 738 \\
\hline
\end{tabular}

\subsection{The Weak Interactions in Intersheet}

Distribution of intersheet nonbonded interactions and change of intersheet hydrogen bonding of cellulose I $\alpha$ are presented in Fig. 5(c) and Table 5. Van der Waals interaction is the highest compared with electrostatic, polar and nonpolar solvation interactions in intersheet of cellulose I $\alpha$ at room temperature from Fig. 5(c). Its average value is $7.5695 \mathrm{Kcal} / \mathrm{mol}$, followed by nonpolar solvation, electrostatic and polar solvation interactions. The average values are $4.2508,3.7727$ and $2.3722 \mathrm{Kcal} / \mathrm{mol}$, respectively [22]. The intersheet Van der Waals energy of cellulose I $\alpha$ increases with the ascending of temperature. The mean value at $550 \mathrm{~K}$ is $7.8438 \mathrm{Kcal} / \mathrm{mol}$, and the electrostatic energy falls into a decline [22]. Polar and nonpolar solvation interactions show the downward trend on the whole. At $550 \mathrm{~K}$, the mean values of Van der Waals, nonpolar, electrostatic and polar solvation interactions are 7.843 8, $4.1613,2.2495$ and $1.9416 \mathrm{Kcal} / \mathrm{mol}$ [22]. So under high temperature Van der Waals interaction is more important than nonpolar solvation, electrostatic, polar solvation interactions. Van der Waals force in cellulose I $\beta$ decreases gradually, accounting for $79.68 \%$ of the total at $550 \mathrm{~K}$, but always keep the highest for intersheet stability of cellulose $\mathrm{I} \beta[21]$.

The intersheet nonbonded interactions of cellulose $\mathrm{I} \alpha$ is higher than that of intrachain and interchain. However, according to Table 5, the hydrogen bond in intersheet is weaker than that of intrachain and interchain. The average occupancy at different temperatures is $0.3345,0.3607$, $0.4153,0.5292,0.4015$ and 0.4567 , respectively, which increases significantly at 400-450 K. In general, the number of hydrogen bonds increased gradually. At $300 \mathrm{~K}$, there are 99 hydrogen bondings in intersheet. At $550 \mathrm{~K}$, the number of hydrogen bonds is 359. Just like the trend of interchain, the number of hydrogen bonding obviously increaes. As for the average occupancy, unlike the change of intrachain and ininterchain hydrogen bonding, the intersheet occupancy increases at high temperature. At $550 \mathrm{~K}$, the hydrogen bond occupancy of intrachain, interchain, 
intersheet is $0.6703,0.5507$ and 0.4567 , respectively, which means that under high temperature the intersheet hydrogen bond occupancy is weaker than that of intrachain and interchain. The synergistic effect is reconfirmed for the intersheet hydrogen bonding is weaker than that of intrachain and interchain, while Van der Waals, electrostatic, polar and nonpolar solvation interactions in intersheet are higher than that of intrachain and interchain.

Table 5: The average occupancy and numbers of intersheet hydrogen bonding under different temperature

\begin{tabular}{ccccccc}
\hline & $300 \mathrm{~K}$ & $350 \mathrm{~K}$ & $400 \mathrm{~K}$ & $450 \mathrm{~K}$ & $500 \mathrm{~K}$ & $550 \mathrm{~K}$ \\
\hline average occupancy & 0.3345 & 0.3607 & 0.4153 & 0.5292 & 0.4015 & 0.4567 \\
The number of hydrogen bonding & 99 & 129 & 302 & 384 & 428 & 359 \\
\hline
\end{tabular}

\section{Conclusion}

The weak interaction mechanism of cellulose I $\alpha$ has been elaborated with molecular dynamic simulations. Among the four interactions investigated, polar solvation shows relatively great significance for intrachain stability. While the nonbonded interactions are relatively weak than interchain and intersheet, the occupancy of intrachain hydrogen bonding is obviously strong. The electrostatic interaction contributes most to the stability of the interchain structure, followed by Van der Waals force, non-polar and polar solvent energy at room temperature. At high temperature, Van der Waals force is higher than electrostatic, polar and non-polar interactions. The occupancy of interchain hydrogen bond decreases sharply. Most of the nonbonded interactions are distributed in intersheet, relatively different from cellulose I $\beta$. We elucidate that the weak hydrogen bonding and strong Van der Waals interaction keep the intersheet stability collaboratively by data statistical analysis. It is also found that there is synergistic effect between hydrogen bonding and other weak interactions. The strong hydrogen bonding complement with relatively weak Van der Waals, electrostatic, polar and non-polar solvation interactions, vice versa.

\section{Acknowledgement}

This work is supported by the Scientific Research Project of Hubei Provincial Department of Education under Grants No. Q20141604. We thank the Laboratory of Biomolecular Dynamics, Wuhan Institute of Physics and Mathematics, Chinese Academy of Sciences for computing supporting.

\section{References}

[1] O'SUlLIVAN A C. Cellulose: the structure slowly unravels. Cellulose, 1997, 4(3): 173-207.

[2] Sorieul M, et al. Plant Fibre: Molecular Structure and Biomechanical Properties, of a Complex Living Material, Influencing Its Deconstruction towards a Biobased Composite. Materials (Basel), 2016, 9(8). 
[3] Aabloo A, et al. Studies of crystalline native celluloses using potential energy calculations. Cellulose, 1994, 1(2): 161-168.

[4] Atalla, R H, D L Vanderhart, Native cellulose: a composite of two distinct crystalline forms. Science, 1984, 223(4633): 283-5.

[5] French A D, D P Miller, A Aabloo, Miniature crystal models of cellulose polymorphs and other carbohydrates. International Journal of Biological Macromolecules, 1993, 15(1): 30-6.

[6] Shen T S. Gnanakaran, The stability of cellulose: a statistical perspective from a coarse-grained model of hydrogen-bond networks. Biophysical Journal, 2009, 96(8): 3032-40.

[7] Wada M, et al. X-ray diffraction study on the thermal expansion behavior of cellulose I beta and its high-temperature phase. Polymer Degradation and Stability, 2010, 95(8): 1330-1334.

[8] Lee C M, et al. Hydrogen-Bonding Network and OH Stretch Vibration of Cellulose: Comparison of Computational Modeling with Polarized IR and SFG Spectra. Journal of Physical Chemistry B, 2015, 119(49): 15138-49.

[9] Shishehbor M, et al. A continuum-based structural modeling approach for cellulose nanocrystals (CNCs). Journal of the Mechanics and Physics of Solids, 2018, 111: 308-332.

[10] Sugiyama J, R Vuong, H Chanzy. Electron diffraction study on the two crystalline phases occurring in native cellulose from an algal cell wall. Macromolecules, 2008, 24(14): 4168-4175.

[11] Hardy B J, A Sarko. Molecular dynamics simulations and diffraction-based analysis of the native cellulose fibre: structural modelling of the I- $\alpha$ and I- $\beta$ phases and their interconversion. Polymer, 1996, 37(10): 1833-1839.

[12] Vanderhart D L, R H Atalla. Studies of microstructure in native celluloses using solid-state carbon13 NMR. Macromolecules, 1984, 17(8): 1465-1472.

[13] Michell A J. Second derivative F.t.-i.r. spectra of celluloses I and II and related mono- and oligosaccharides. Carbohydrate Research, 1988, 173(2): 185-195.

[14] Yamamoto H, F Horii, H Odani. Structural changes of native cellulose crystals induced by annealing in aqueous alkaline and acidic solutions at high temperatures. Macromolecules, 1989, 22(10): 4130-4132.

[15] Nishiyama Y, P Langan, H Chanzy. Crystal structure and hydrogen-bonding system in cellulose Ibeta from synchrotron X-ray and neutron fiber diffraction. Journal of the American Chemical Society, 2002, 124(31): 9074-82.

[16] Nishiyama Y, P Langan, H Chanzy. Crystal structure and hydrogen-bonding system in cellulose Ibeta from synchrotron X-ray and neutron fiber diffraction. Journal of the American Chemical Society, 2003, 125(47): 14300-6.

[17] Jones D W. Crystalline modifications of cellulose. Part III. The derivation and preliminary study of possible crystal structures. Journal of Polymer Science, 1958, 32(125): 371-394.

[18] Heiner A P, J Sugiyama, O Teleman. Crystalline Cellulose I-Alpha And I-Beta Studied by MolecularDynamics Simulation. Carbohydrate Research, 1995, 273(2): 207-223.

[19] Nishiyama Y. Molecular interactions in nanocellulose assembly. Physical and Engineering Sciences, 2018, 376(2112).

[20] French A D, D P Miller, A Aabloo. Miniature crystal models of cellulose polymorphs and other carbohydrates. International Journal of Biological Macromolecules, 1993, 15(1): 30-6.

[21] Jiang X W, H H Zhang, A H Zhong. Revealing the Interaction Mechanism Stabilizing Crystalline Cellulose I $\beta$ by Molecular Dynamics Simulations. Journal of Fiber Bioengineering and Informatics, 2017, 10(3): 141-154.

[22] Zhang H H, X W Jiang, Y Chen. The Van der Waals and Electrostatic Interaction Analysing of Crystalline Cellulose I $\alpha$ Based on Molecular Dynamics. Textile Bioengineering and Informatics Symposium Proceedings, 2018: 969-975. 
[23] Zhang Q, et al. A molecular dynamics study of the thermal response of crystalline cellulose $\mathrm{I} \beta$. Cellulose, 2011, 18(2): 207-221.

[24] ] Kirschner K N, et al. GLYCAM06: a generalizable biomolecular force field. Carbohydrates. Journal of Computational Chemistry, 2008, 29(4): 622-55.

[25] Matthews J F, et al. High-temperature behaviour of cellulose I. Journal of Physical Chemistry B, 2011, 115(10): 2155-66.

[26] Vangara R, F van Swol, D N Petsev. Ionic solvation and solvent-solvent interaction effects on the charge and potential distributions in electric double layers. Journal of Chemical Physics, 2017, 147(21): 214704.

[27] Chen P, et al. Diversity of potential hydrogen bonds in cellulose I revealed by molecular dynamics simulation. Cellulose, 2014, 21(2): 897-908.

[28] Tanaka F, K Okamura. Characterization of cellulose molecules in bio-system studied by modelling methods. Cellulose, 2005, 12(3): 243-252.

[29] Eichhorn S J, G R Davies. Modelling the crystalline deformation of native and regenerated cellulose. Cellulose, 2006, 13(3): 291-307.

[30] Shuang Huang. Research on the Micro mechanism of native cellulose like cotton and linen fibres based on molecular simulation. 2014, Donghua University.

[31] And K M, L Heux. Molecular Dynamics Simulations of Bulk Native Crystalline and Amorphous Structures of Cellulose. Journal of Physical Chemistry B, 2003, 107(10): 2394-2403.

[32] Bergenstrahle M, L A Berglund, K. Mazeau. Thermal response in crystalline I beta cellulose: A molecular dynamics study. Journal of Physical Chemistry B, 2007, 111(30): 9138-9145. 